\title{
Crenças de pessoas com insuficiência cardíaca relacionadas à ingesta de sal
}

\author{
Beliefs of people with salt intake-related heart failure \\ Creencias de las personas con insuficiencia cardíaca relacionadas con la ingesta de sal
}

\author{
Mailson Marques de Sousa'; Bernadete de Lourdes André Gouveia"; Taciana da Costa Farias Almeida"l'; \\ Maria Eliane Moreira Freire ${ }^{\mathrm{IV}}$; Simone Helena dos Santos Oliveira ${ }^{v}$
}

\begin{abstract}
RESUMO
Objetivo: analisar as crenças comportamentais, normativas e de controle relacionadas à redução do consumo de sal na dieta de pessoas com insuficiência cardíaca (IC). Método: estudo com abordagem quantiqualitativa norteado pela Theory of Planned Behavior, realizado mediante entrevista individual com 26 pessoas com IC em seguimento ambulatorial, no período de janeiro a fevereiro de 2018, no município de João Pessoa. Foram aplicadas análises estatística e de conteúdo. Resultados: sobressaíram a manutenção da saúde e diminuição do apetite como crenças comportamentais positivas e negativas, respectivamente. Com relação às crenças normativas, cônjuges, filhos e irmãos apresentaram-se como referentes positivos que exercem maior influência na redução de sal. Quanto às crenças de controle, o preparo das refeições pela esposa facilita e a perda do sabor dos alimentos dificulta a execução do comportamento esperado. Conclusão: a análise das crenças permitiu elucidar aquelas que precisam ser fortalecidas e atenuadas frente à redução de sal para controle clínico da IC.

Descritores: Insuficiência cardíaca; dieta hipossódica; comportamento; teoria social.
\end{abstract}

\section{ABSTRACT}

Objective: to examine behavioral, normative, and control-related beliefs about reducing dietary salt intake in people with heart failure (HF). Method: this quantitative and qualitative study, guided by the Theory of Planned Behavior, was conducted from January to February 2018, through individual interviews of 26 people with HF in outpatient follow-up in the city of João Pessoa. Statistical and content analyses were applied. Results: maintenance of health and decreased appetite stood out, respectively, as positive and negative behavioral beliefs. Normative beliefs featured spouses, children and siblings as positive referents exerting greatest influence on salt reduction. Control-related beliefs included wife's preparation of meals making it easier, and tasteless food harder, to behave as expected. Conclusion: by examining beliefs it was possible to elucidate those needing to be strengthened or attenuated with a view to reducing salt intake for clinical control of HF.

Descriptors: Heart failure; hyposodic diet; behavior; social theory.

\section{RESUMEN}

Objetivo: analizar las creencias conductuales, normativas y de control relacionadas con la reducción de ingesta de sal en la dieta de personas con insuficiencia cardíaca (IC). Método: un estudio con enfoque cuantitativo y cualitativo guiado por la Theory of Planned Behavior, realizado a través de una entrevista individual junto a 26 personas con insuficiencia cardíaca en seguimiento ambulatorio, de enero a febrero de 2018, en la ciudad de João Pessoa. Se aplicaron análisis estadísticos y de contenido. Resultados: el mantenimiento de la salud y la disminución del apetito se destacaron como creencias conductuales positivas y negativas, respectivamente. Con respecto a las creencias normativas, los cónyuges, hijos y hermanos se mostraron como referentes positivos que ejercen una mayor influencia en la reducción de sal. En cuanto a las creencias de control, la preparación de las comidas por parte de la esposa facilita y la pérdida del sabor de los alimentos dificulta la realización de la conducta esperada. Conclusión: el análisis de creencias permitió mostrar las que necesitan ser fortalecidas y atenuadas ante la reducción de sal para el control clínico de la insuficiencia cardíaca.

Descriptores: Insuficiencia cardíaca; dieta hiposódica; comportamiento; teoría social.

\section{INTRODUÇÃO}

Entre as medidas não farmacológicas prescritas na prática clínica para pessoas com insuficiência cardíaca (IC), a restrição no consumo de sal/sódio é amplamente orientada nos diferentes níveis de assistência à saúde ${ }^{1}$. Contudo, as evidências sobre os seus benefícios e desfechos adversos ainda permanecem inconclusivas em razão da variedade de protocolos, amostras e métodos de análises, sendo oportuno realizar investigações adicionais de alto impacto ${ }^{2}$.

Diretrizes nacionais ${ }^{3}$ e internacionais ${ }^{4}$ recomendam orientar e educar as pessoas com IC a diminuírem a ingestão de alimentos com alto teor de sal em suas dietas, sendo essa indicação individualizada para as condições clínicas, por exemplo, a sintomatologia apresentada e terapêutica farmacológica utilizada, em especial o uso de diuréticos a fim de minimizar quadros de hipervolemia.

'Enfermeiro. Mestre. Aluno do curso de doutorado. Universidade Federal da Paraíba. João Pessoa, Brasil. E-mail: mailson ms@hotmail.com "Enfermeira. Mestre. Aluna do curso de doutorado. Universidade Federal da Paraíba. João Pessoa, Brasil. E-mail: bernagouveia46@gmail.com I'Enfermeira. Mestre. Aluna do curso de doutorado. Universidade Federal da Paraíba. João Pessoa, Brasil. E-mail: tacianacfalmeida@gmail.com IVEnfermeira. Doutora. Professora Adjunta. Universidade Federal da Paraíba. João Pessoa, Brasil. E-mail: enf.elimoreirafreire@gmail.com

vEnfermeira. Pós-Doutora. Professora Titular. Universidade Federal da Paraíba. João Pessoa, Brasil. E-mail: simonehsoliveira@gmail.com 
A literatura científica aponta baixa adesão de pessoas com IC ao consumo reduzido de sal na dieta ${ }^{5,6}$. A falta de conhecimento sobre como fazer uma dieta com baixo teor de sal, a interferência e socialização familiar no preparo e ingestão de alimentos com alto teor de sal, a dificuldade de escolher refeições fora de casa, bem como a baixa palatabilidade dos alimentos preparados com pouco sal são fatores descritos para a não adesão ao seguimento da dieta $^{7}$. Assim, torna-se relevante que o desenvolvimento de tecnologias de cuidado que favoreçam a adesão às medidas protetoras da saúde sejam ancoradas em modelos teóricos consistentes, que possam explicar os determinantes que influenciam um comportamento específico.

Nesse sentido, destaca-se a Theory of Planned Behavior (TPB) utilizada para compreender, explicar e predizer o comportamento humano em grupos e contextos específicos. Para a teoria, o comportamento é determinado pela intenção (motivação) para agir (efetivar o comportamento) e a percepção de controle sobre o comportamento. A intenção é formada por três fatores: a atitude, a norma subjetiva e o controle comportamental percebido ${ }^{8}$.

Estudos internacionais demonstram a preocupação em identificar os fatores determinantes para a execução do comportamento investigado ${ }^{5,9}$. No Brasil, as pesquisas com o escopo de deslindar as crenças de pessoas com IC à luz da TPB são incipientes. Portanto, torna-se primordial identificar as crenças que antecedem os determinantes do comportamento. Além disso, a elucidação de crenças se constitui como a primeira etapa do referencial teórico para o desenvolvimento de instrumentos de medida e planejamento de tecnologias de cuidado, direcionadas à motivação do comportamento na adesão às medidas de promoção e proteção à saúde.

Nesse contexto, definiu-se como comportamento de interesse para o estudo a redução do consumo de sal na dieta nos próximos 30 dias, o que contempla os elementos de alvo, ação, contexto e tempo, de acordo com os pressupostos teóricos adotados, para compreender os aspectos positivos e negativos, facilidades e dificuldades para a sua consecução, concorrendo para avanços favoráveis nos desfechos clínicos dessa população.

Diante do exposto, o objetivo deste estudo foi analisar as crenças comportamentais, normativas e de controle relacionadas à redução do consumo de sal na dieta de pessoas com IC.

\section{REFERENCIAL TEÓRICO}

De acordo com a TPB, para predizer a intenção comportamental, a partir dos conceitos de atitude, norma subjetiva e controle comportamental percebido, torna-se essencial a elucidação das crenças salientes em relação ao fenômeno de interesse. Crenças salientes são as mais frequentes, isto é, mencionadas mais vezes e originadas a partir de um universo e assunto comum, quando o indivíduo é abordado por meio de questões abertas sobre o comportamento estudado ${ }^{8}$.

Nesse contexto, o primeiro antecedente, a atitude, é composto pelas crenças comportamentais, que se relacionam com a favorabilidade do sujeito quanto à realização ou não do comportamento e as avaliações das consequências positivas ou negativas para a execução do comportamento em questão ${ }^{8}$.

O segundo antecedente, a norma subjetiva, refere-se à influência social, ou seja, a pressão social percebida, constituída pelas crenças normativas que refletem na percepção do indivíduo sobre as opiniões dos seus referentes sociais acerca da realização ou não do comportamento, bem como na motivação individual para concordar com os referentes sociais ${ }^{8}$.

O terceiro antecedente, controle comportamental percebido, é constituído pelas crenças de controle; consiste nas percepções do indivíduo em relação à capacidade de executar ou não o comportamento, bem como à presença de fatores que podem facilitar ou dificultar o seu desempenho. Entre esses fatores estão os de controle interno (informações, deficiências pessoais, habilidades, emoções) e externos (oportunidades, dependência de outros, barreiras) ${ }^{8}$.

\section{METODOLOGIA}

Trata-se de estudo descritivo, com abordagem quantiqualitativa, desenvolvido em duas instituições públicas de saúde do Município de João Pessoa/PB, no período de janeiro a fevereiro de 2018.

Seguindo os preceitos do referencial teórico adotado, não há exigência quanto ao número de sujeitos para elucidar as crenças salientes acerca de um comportamento de interesse. Todavia, consoante o critério de saturação dos dados recomendado pela $\mathrm{TPB}^{8,10}$ para levantamento de crenças, em que novas informações não fossem mais obtidas, neste estudo, a saturação das informações foi alcançada em declarações de 26 respondentes.

Dessa forma, a amostra foi constituída por 26 participantes. Utilizou-se amostra não probabilística, por conveniência, adotando-se como critérios de inclusão para seleção da amostra: pessoas com diagnóstico de IC confirmado em prontuário, com idade $\geq 18$ anos, de ambos os sexos, independente da etiologia, categorizados nas classes funcionais I-III da New York Heart Association (NYHA) ${ }^{3}$, possuir registro da fração de ejeção do ventrículo esquerdo avaliada por meio de 
ecocardiograma transtorácico nos últimos seis meses e em seguimento ambulatorial. Excluíram-se pessoas em primeira consulta por, possivelmente, não estarem ainda devidamente cientes das medidas do manejo não farmacológico para controle clínico da IC, e que apresentassem déficit de cognição verbal informado em prontuário.

A coleta de dados ocorreu por meio de entrevista individual gravada em áudio, realizada após informações sobre o estudo aos participantes e obtenção de sua anuência por escrito (Termo de Consentimento Livre e Esclarecido), norteada por dois instrumentos. O primeiro instrumento foi um questionário contendo questões sociodemográficas e clínicas para a caracterização dos participantes. O segundo instrumento, um formulário estruturado com seis questões abertas sob o formato de respostas livres, a fim de suscitar as crenças comportamentais, normativas e de controle sobre a redução do consumo de sal na dieta nos próximos 30 dias. Tal instrumento foi construído em consonância com os pressupostos teóricos da TPB ${ }^{8,10}$, cujo conteúdo fora avaliado por três pesquisadores no Grupo de Estudos e Pesquisa em Doenças Crônicas da Universidade Federal da Paraíba. Em seguida, foi realizado teste-piloto com 10 pessoas com IC que evidenciou adequação dos instrumentos empregados na técnica de coleta de dados para a população-alvo, permitindo a continuidade do estudo.

Para a investigação das crenças comportamentais positivas e negativas, os questionamentos foram: Na sua opinião, quais as vantagens de você reduzir o consumo de sal na dieta nos próximos 30 dias?; na sua opinião, quais as desvantagens de você reduzir o consumo de sal na dieta? Para crenças normativas, positivas e negativas: quais as pessoas importantes, para você, consideram que você deve reduzir o consumo de sal na dieta?; quais as pessoas importantes, para você, consideram que você não deve reduzir o consumo de sal na dieta? E, para avaliar as crenças de controle: quais fatores facilitam que você reduza o consumo de sal na dieta nos próximos 30 dias?; quais fatores dificultam que você reduza o consumo de sal na dieta nos próximos 30 dias?

Com relação à análise dos dados, foi aplicada a estatística descritiva. Para variáveis contínuas, calcularam-se média e desvio padrão. As variáveis categóricas foram analisadas por meio do cálculo das frequências absoluta e percentual. Quanto às crenças relacionadas à redução do consumo de sal na dieta, estas foram submetidas à análise de conteúdo, de acordo com as recomendações propostas pela TPB. Primeiro, procedeu-se à leitura das transcrições das falas dos participantes, seguida da seleção das respostas para categorização, pelo critério de similitude e pela contagem da frequência de sua emissão. Adotou-se como critério que as crenças modais salientes deveriam representar índice $\geq 75 \%$ do total das respostas emitidas ${ }^{8,10}$. A partir deste critério, crenças com três emissões ou mais compuseram o jogo modal. Utilizaram-se os construtos determinados pela TPB, crenças comportamentais, crenças normativas e crenças de controle, para agrupamento das respostas ${ }^{8,10}$.

O estudo foi aprovado pelo Comitê de Ética em Pesquisa sob o Parecer no 2.406.616/2017 e CAEE: 79911717.0.0000.5183.

\section{RESULTADOS}

A amostra foi constituída por 26 pessoas com IC, com idade média de $61,27 \pm 11,80$, com variação de 35 a 82 anos. Dentre os participantes, 14 (53,8\%) eram do sexo masculino, 15 (57,7\%) se autodeclararam não brancos, 16 (61,5\%) viviam casados ou em união estável. A escolaridade média correspondeu a 4,81 anos de estudo, 25 (92,2\%) apresentavam-se economicamente inativos e 23 (88,5\%) possuíam renda familiar entre um e dois salários mínimos.

Com relação às características clínicas, verificou-se que a maioria, $15(57,7 \%)$, se encontrava na classe funcional II, segundo os critérios da NYHA. Quanto à etiologia da IC, 18 (69,2\%) possuíam miocardiopatia de origem não isquêmica. No que concerne a comorbidades associadas à IC, 13 (35,1\%) apresentavam hipertensão arterial sistêmica e 9 (24,3\%),

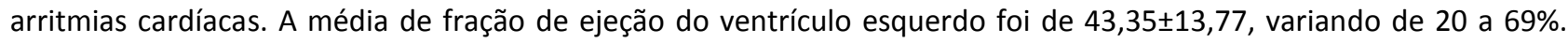
Diuréticos e betabloqueadores foram os principais medicamentos utilizados, mencionados por 21 (38,2\%) e 19 (34,5\%) dos participantes, respectivamente.

A respeito da análise sobre crenças comportamentais, quanto às vantagens e desvantagens em reduzir o consumo de sal na dieta, evidencia-se maior frequência para a manutenção da saúde e diminuição do apetite, respectivamente, conforme mostra a Tabela 1.

No que tange às crenças normativas, ou seja, as pessoas que são importantes para os investigados com IC e consideram que elas devem reduzir o consumo de sal na dieta, destacaram-se cônjuges como referentes sociais positivos, segundo a Tabela 2.

Concernente aos referentes negativos, dos 26 participantes da pesquisa, 21 (80,7\%) referiram não haver pessoas que achassem importante não reduzir o consumo de sal na dieta nos próximos 30 dias. Observaram-se duas ocorrências para familiares, uma emissão para esposo, irmão, e o próprio participante. Considerando o critério adotado para o estudo de incluir como crenças salientes aquelas emitidas por pelo menos três respondentes, identificou-se ausência de referentes negativos. 
TABELA 1: Frequência das crenças comportamentais positivas e negativas sobre reduzir o consumo de sal na dieta. João Pessoa, PB, Brasil, 2018.

\begin{tabular}{lclc}
\hline \multicolumn{1}{c}{ Vantagens } & $\mathbf{f}(\%)$ & \multicolumn{1}{c}{ Crenças comportamentais } \\
& $22(81,4)$ & Diminui o apetite & $\mathbf{f ( \% )}$ \\
\hline Mantém a saúde & $4(14,8)$ & Mudança no gosto dos alimentos & $8(40,0)$ \\
Evita a piora dos sintomas & $7(35,0)$ \\
Idiossincráticas & $1(3,8)$ & Hábito de usar o sal & $3(15,0)$ \\
& & Reclamação dos familiares & $1(5,0)$ \\
Total & & Cozinhar separadamente & $1(5,0)$ \\
CCMS $\left.^{*}\right)$ & $27(100,0)$ & & $20(100,0)$ \\
\hline
\end{tabular}

${ }^{(*)}$ Crenças comportamentais modais salientes

TABELA 2: Frequência das crenças normativas/referentes positivao sobre reduzir o consumo de sal na dieta. João Pessoa, PB, Brasil, 2018.

\begin{tabular}{lc}
\hline Referentes positivos & $\mathbf{f ~ ( \% )}$ \\
\hline Esposa(o) & $15(48,5)$ \\
Filhos(as) & $9(29,0)$ \\
Irmãos(ãs) & $3(9,6)$ \\
Família & $2(6,5)$ \\
Vizinhos & $1(3,2)$ \\
Sobrinhos & $1(3,2)$ \\
Total & $31(100,0)$ \\
CNMS(*) & $27(87,1)$ \\
\hline${ }^{(*)}$ Crenças normativas modais salientes
\end{tabular}

Quanto às crenças de controle, que avaliam as facilidades e barreiras à execução do comportamento, o preparo das refeições pela esposa facilita e a perda do sabor dos alimentos dificulta, de acordo com a Tabela 3.

TABELA 3: Frequência das crenças de controle positivas e negativas sobre reduzir o consumo de sal na dieta - João Pessoa, PB, Brasil, 2018 .

\begin{tabular}{|c|c|c|c|}
\hline \multicolumn{4}{|c|}{ Crenças de controle } \\
\hline $\begin{array}{c}\text { Facilidades } \\
\end{array}$ & n (\%) & Dificuldades & n (\%) \\
\hline Preparo das refeições pela esposa & $8(42,1)$ & Perda do sabor dos alimentos & $10(62,5)$ \\
\hline Preparo das refeições separadamente & $4(21,0)$ & $\begin{array}{l}\text { Falta de controle na escolha de } \\
\text { alimentos para o preparo das refeições }\end{array}$ & $4(25,0)$ \\
\hline Família ajuda a lembrar & $4(21,0)$ & Família gostar de usar sal & $2(12,5)$ \\
\hline Gostar da comida com pouco sal & $1(5,3)$ & & \\
\hline Substituir o sal por ervas e temperos & $1(5,3)$ & & \\
\hline Idiossincráticas & $1(5,3)$ & & \\
\hline Total & $19(100)$ & & $16(100)$ \\
\hline $\mathrm{CCMS}^{(*)}$ & $16(84,1)$ & & $14(87,5)$ \\
\hline
\end{tabular}

${ }^{(*)}$ Crenças de controle modais salientes

\section{DISCUSSÃO}

Em relação à análise das crenças comportamentais, de acordo com a TPB, pode-se afirmar que, por meio das crenças, uma pessoa pondera as consequências de uma futura ação, em uma dimensão de favorabilidade ou desfavorabilidade. 
Identificaram-se como vantagens manter a saúde e evitar a piora dos sintomas da IC. Assim, embora os achados sejam coerentes, verifica-se percepção limitada dos benefícios da redução do consumo de alimentos com alto teor de sal para a saúde, uma vez que apenas $14,8 \%$ dos participantes associaram os benefícios ao controle da sintomatologia da IC.

Esse fato pode estar relacionado com a falta de conhecimento, finalidade e efeitos da redução do consumo de sal na fisiopatologia da doença. Sabe-se que o excesso na ingestão de sódio provoca retenção e acúmulo de líquidos, desencadeando alterações no controle do volume extracelular, que produzem quadros de descompensação e solicitam atendimento clínico imediato ${ }^{11}$.

Concordante com esses achados, pesquisa nacional que analisou o conhecimento de pessoas com IC sobre a doença identificou que este é fundamentado em experiências individuais, sem embasamento científico, mas por meio da percepção comum apreendida no manejo diário da $\mathrm{IC}^{12}$. Com isso, torna-se primordial que estratégias educativas delineadas pela equipe multidisciplinar sejam testadas e implementadas na assistência oferecida a essa população, a fim de clarificar o processo saúde/doença, favorecendo uma visão ampliada da gestão de autocuidado e qualidade de vida.

Entre as crenças comportamentais negativas, diminuição do apetite, mudança no gosto dos alimentos e hábito de usar sal apresentaram-se como salientes. Nota-se que as mudanças ocorridas na palatabilidade dos alimentos associadas ao hábito alimentar de uso do sal no preparo das refeições configuram-se como aspectos negativos para a redução do consumo de sal na dieta.

Assim, observa-se coerência em relação às crenças emitidas sobre as desvantagens de reduzir o consumo de sal. Esses achados remetem a reflexões de contexto cultural no que tange à culinária local, que é fortemente influenciada pela preferência de alimentos ricos em sódio. Além disso, o hábito de usar condimentos industrializados no preparo e cozimento dos alimentos, bem como o uso de sal à mesa para acentuar o sabor das refeições remetem à sensação de prazer e bem-estar ${ }^{13}$. A prática dessas ações necessita ser reavaliada para a mudança necessária do estilo de vida, de forma a favorecer a manutenção da estabilidade clínica da IC.

Em pessoas com IC, a ativação do sistema renina-angiotensina aldosterona induz estímulos neuroendócrinos a mudanças gustativas, pela baixa concentração de sódio no organismo, favorecendo a percepção de um paladar satisfatório e prazeroso ao se ingerir dietas com alto teor de sal ${ }^{13}$. Condizente com essa afirmativa, um estudo de coorte prospectivo, que analisou a palatabilidade percebida de alimentos com diferentes concentrações de sal em pessoas com IC clinicamente compensados, evidenciou preferência significativamente maior para refeições com concentração elevada de sal, quando comparados a indivíduos saudáveis $(p<0,001)^{14}$

Sendo assim, os resultados encontrados abrem perspectivas para o desenvolvimento de tecnologias de cuidado, com o fito de reverter crenças negativas em positivas, a fim de fortalecer a adesão às medidas protetoras da saúde. Desse modo, comunicações persuasivas no formato audiovisual, baseadas nas crenças das pessoas com IC sobre o tema, podem ser testadas como estratégia de intervenção no âmbito individual e/ou coletivo para influenciar mudanças de crenças, atitudes e comportamento frente à redução do consumo de sal. Comunicações de mídia para a redução do consumo de sal configuram-se como estratégias indicadas pela Organização Mundial de Saúde para promoção, controle e manutenção da estabilidade clínica de clientes com doenças crônicas cardiovasculares a ser alcançada até $2025^{15}$.

No tocante às crenças normativas, os referentes sociais positivos modais salientes foram cônjuges, filhos e irmãos. Pelos resultados encontrados, observa-se que pessoas de convívio diário próximo são os referentes mais importantes que podem exercer influência na motivação para o alcance do comportamento esperado. Por isso, compreende-se que o apoio familiar pode auxiliar no incremento das mudanças alimentares necessárias ao manejo clínico da IC.

Cabe destacar que, no processo de enfrentamento de uma doença crônica como a IC, a construção de redes sociais de apoio colabora para o sucesso da terapêutica instituída. Incorporar novos hábitos alimentares requer incentivo e compreensão do núcleo social em que se convive ${ }^{16,17}$. Presume-se que experiências compartilhadas favoreçam um melhor entendimento e desenvolvimento de habilidades que promovam a prática de autocuidado e, por conseguinte, a qualidade de vida. Portanto, é oportuno refletir sobre a estruturação de redes de apoio com a integração de membros da família, vizinhos, amigos, que poderão compartilhar sentimentos e experiências no conviver, cuidar e potencializar o autocuidado de pessoas com IC no que concerne ao seguimento da terapêutica prescrita e, especialmente, na redução do consumo de sal na dieta.

Nota-se que, entre as crenças normativas, não emergiram referentes sociais negativos. Nesse sentido, considerase que as pessoas importantes para os entrevistados apoiavam a redução do consumo de sal. Desse modo, reafirma-se a importante participação familiar na motivação de pessoas com IC a consumirem uma dieta com baixo teor de sal, contribuindo para minimizar avanços deletérios ao miocárdio e possíveis internações hospitalares indesejáveis.

$\mathrm{Na}$ amostra investigada, os profissionais de saúde não foram mencionados como referentes positivos ou negativos, dado que se mostra preocupante, sugerindo fragilidades no vínculo deles com os usuários do serviço, uma 
vez que tais profissionais são considerados agentes promotores no fomento à redução do consumo de sal na população, meta definida pela Organização Mundial de Saúde e assumida pelo Brasil no plano de enfrentamento para controle das doenças crônicas não transmissíveis ${ }^{18}$.

Assim, sugere-se que os profissionais invistam em estratégias de autocuidado para o fortalecimento do vínculo com os usuários nos diversos cenários de assistência à saúde, a fim de se tornarem referentes positivos com potencial para favorecer o conhecimento sobre condições de saúde, bem como o suporte necessário na solução de problemas, concorrendo para melhor gestão terapêutica ${ }^{19}$.

No que se refere às crenças de controle, evidencia-se que o preparo das refeições pela esposa, preparar as refeições separadamente e a família ajudar a lembrar são crenças salientes concernentes às facilidades para a redução do consumo de sal. No entanto, verifica-se relação de dependência entre os participantes e os seus referentes sociais no que diz respeito ao alcance do comportamento esperado.

Tais achados remetem às reflexões de contexto histórico e social quando determinam que a esposa seja responsável pela administração e manutenção de atividades domésticas, dentre elas o preparo da alimentação. Ao longo das últimas décadas, embora a coparticipação masculina venha aumentando na dinâmica de tarefas domésticas, torna-se importante que estratégias sejam implementadas para os referentes responsáveis pelo preparo das refeições, com intuito de clarificar aspectos da dietoterapia de pessoas com IC e da importância da redução do consumo de sal não somente voltado ao controle destes casos, mas para a saúde cardiovascular da família.

Um estudo multicêntrico internacional, cujo objetivo foi examinar a adesão familiar a uma dieta com baixo teor de sódio e seu efeito na adesão de pessoas com IC, concluiu que viver com um cônjuge ou outro membro da família melhorou a aderência do paciente a uma dieta com baixo teor o sódio. Os autores evidenciam que os pacientes, cujos membros da família seguiram a dieta recomendada, apresentaram menor excreção de sódio urinário $(p=0,003)$ e foram 1,6 vezes mais susceptíveis à adesão alimentar com baixo teor de sódio $(p=0,035)^{20}$.

Assim, reforça-se o papel da família como adjuvante na adesão às medidas de manejo não farmacológicas recomendadas para a estabilidade clínica dos clientes com IC. Sugere-se que ações com abordagem nutricional sejam desenvolvidas para todo o arranjo familiar, considerando condições socioeconômicas e clínicas, a fim de identificar habilidades e potencialidades para a execução de uma dieta com menor quantidade de alimentos com alto teor de sal e maior ingesta de alimentos saudáveis.

Quanto às crenças de controle, perda do sabor dos alimentos e falta de controle no preparo das refeições foram salientes. Estudos apontam que comorbidades associadas à IC, deficiência de vitaminas, fatores de risco nocivos à saúde, como tabagismo, e a terapêutica diurética utilizada no manejo farmacológico da doença podem alterar a percepção de palatabilidade dos alimentos, face ao déficit de eletrólitos, que pode influenciar a sensação de perda de sabor dos alimentos e a rejeição à uma dieta com baixo teor de sal ${ }^{13,21}$.

Ademais, a restrita variedade alimentar dificulta o controle de alimentos no preparo de refeições, especialmente para pacientes que consomem refeições fora de casa ${ }^{7,22}$. A presença de alimentos industrializados, ultraprocessados e o aumento expressivo das redes de fast-food são aspectos que facilitam o acesso e aquisição de refeições com alto teor de sal.

Diante desses achados, considera-se pertinente a implementação de intervenções educativas que busquem ampliar o conhecimento e entendimento dessa população sobre manutenção do sabor e preparo dos alimentos. Sugerese a substituição de temperos industrializados que possuem alto teor de sódio para sua conservação, pelo uso de ervas, temperos naturais e especiarias, a fim de contribuir para realçar a palatabilidade dos alimentos, proporcionando sensação de bem-estar e prazer pelo seu consumo.

Como limitação do estudo, pode-se apontar a carência de respostas frente à compreensão do fenômeno investigado. Por isso, são necessárias novas pesquisas conduzidas nas diferentes regiões do país, tendo em vista a pluralidade cultural e de hábitos alimentares, para ratificar, expandir ou refutar os achados ilustrados nesta investigação, pois este é um comportamento humano sujeito às influências do meio sociocultural em que vivem as pessoas, não sendo possível a generalização dos achados.

Para a prática de enfermagem, este estudo suscita reflexões quanto à necessidade de enfatizar mais a importância da redução do consumo de sal nas ações educativas direcionadas à pessoa com doença cardiovascular. Os resultados obtidos subsidiarão o desenvolvimento de tecnologias de cuidado de cunho persuasivo, com o escopo de fortalecer crenças positivas e reverter crenças negativas. Além do mais, subsidiarão a construção de escala psicométrica para mensurar os fatores determinantes do comportamento de redução do consumo de sal na dieta. 


\section{CONCLUSÃO}

Os resultados encontrados nesta investigação revelaram as crenças comportamentais, normativas e de controle de pessoas com IC que devem ser fortalecidas e atenuadas frente à redução do consumo de sal na dieta, que apresenta vantagens para a manutenção da saúde e evitar a piora dos sintomas da IC. Nota-se que pessoas de convívio familiar exercem forte influência para a redução do consumo de sal, bem como destacam-se como membros facilitadores para o alcance do comportamento esperado. Do mesmo modo, foi identificado que alterações na palatabilidade dos alimentos se constituem como desvantagens e barreiras para a adesão a uma dieta com baixo teor de sal.

\section{REFERÊNCIAS}

1. Gonçalves FG, Albuquerque DC. Health education of patients with heart failure. Rev. enferm. UERJ. [Internet] 2014 [cited 2019 Jun 10]; 22(3):422-8. Available from: https://www.e-publicacoes.uerj.br/index.php/enfermagemuerj/article/view/13769/10514

2. Mahtani KR, Heneghan C, Onakpoya, Tierney S, Aronson JK, Roberts N. Reduced salt intake for heart failure: a systematic review. JAMA intern. med. [Internet] 2018 [cited 2019 Jun 10]; 178(12):1693-700. Available from: https://jamanetwork.com/journals/jamainternalmedicine/article-abstract/2712563

3. Comitê Coordenador da Diretriz de Insuficiência Cardíaca. Diretriz Brasileira de Insuficiência Cardíaca Crônica e Aguda. Arq. bras. cardiol. [Internet] 2018 [cited 2019 Jun 10]; 111 (3): 436-539. DOI: http://dx.doi.org/10.5935/abc.20180190

4. Yancy CW, Jessup M, Bozkurt B, Butler J, Casey DE Jr, Drazner MH, et al. 2013 ACCF/AHA guideline for the management of heart failure: a report of the American College of Cardiology Foundation/American Heart Association task force on practice guidelines. J. Am. Coll. Cardiol. [Internet], 2013 [cited 2019 Jun 10]; 62(16):e147-239. Available from: http://www.onlinejacc.org/content/accj/70/6/776.full.pdf

5. Wu J-R, Lennie TA, Dunbar SB, Pressler SJ, Moser DK. Does the Theory of Planned Behavior predict dietary sodium intake in patients with heart failure? West .J. Nurs. Res. [Internet] 2017 [cited 2019 Jun 10]; 39(4):568-81. DOI: https://doi.org/10.1177/0193945916672661

6. Rong $X$, Peng Y, Yu HP, Li D. Cultural factors influencing dietary and fluid restriction behavior: perceptions of older chinese patients with heart failure. J. clin. nurs. [Internet] 2017 [cited 2019 Jun 10];26(5-6):717-26. DOI: https://doi.org/10.1111/jocn.13515

7. Bentley B, De Jong MJ, Moser DK, Peden AR. Factors related to nonadherence to low sodium diet recommendations in heart failure patients. Eur. j. cardiovasc. nurs. [Internet] 2005 [cited 2019 Jun 10]; 4(4):3316. DOI: https://doi.org/10.1016/j.ejcnurse.2005.04.009

8. Ajzen I. The theory of planned behavior. Organ. Behav. Hum. Decis. Process. [Internet] 1991 [cited 2019 Jun 10]; 50:179-211. DOI: https://doi.org/10.1016/0749-5978(91)90020-T

9. Welsh D, Lennie TA, Marcinek R, Biddle MJ, Abshire D, Bentley B, et al. Low-sodium diet self-management intervention in heart failure: pilot study results. Eur. j. cardiovasc. nurs. [Internet] 2013 [cited 2019 Jun 10];12(1):87-95. DOI: https://doi.org/10.1177/1474515111435604

10. Francis C, Francis AJJ, Eccles MP, Johnston M, Walker A, Grimshaw J, et al. Constructing questionnaires based on the theory of planned behaviour: a manual for health services researchers. City Research Online City:University of London Institutional Repositor; 2004.

11. Song EK, Moser DK, Kang SM, Lennie TA. Self-reported adherence to a low-sodium diet and health outcomes in patients with heart failure. J. cardiovasc. nurs. [Internet] 2016 [cited 2019 Jun 10]; 31(6):529-34. Available from: https://www.ncbi.nlm.nih.gov/pubmed/26296246

12. De Freitas MTS, Püschel VAA. Heart failure: expressions of personal knowledge about the disease. Rev. Esc. Enferm. USP. [Internet], 2013 [cited 2019 Jun 10]; 47(4):922-6. DOI: http://dx.doi.org/10.1590/S0080-623420130000400021

13. Wessler JD, Hummel SL, Maurer MS. Dietary interventions for heart failure in older adults: Re-emergence of the hedonic shift. Prog. cardiovasc. dis. [Internet] 2014 [cited 2019 Jun 10]; 57(2):160-7. DOI: https://doi.org/10.1016/j.pcad.2014.03.007

14. De Souza JT, Matsubara LS, Menani JV, Matsubara BB, Johnson AK, De Gobbi JIF. Higher salt preference in heart failure patients. Appetite. [Internet] 2012 [cited 2019 Jun 10]; 58(1):418-23. DOI: https://doi.org/10.1016/j.appet.2011.09.021

15. WHO. Global status report on noncommunicable diseases 2014. Geneve(Swi) World Health.Organization; 2014.

16. Menezes HF, Rosas AMMTF, Camacho ACLF, Souza FS, Rodrigues BMRD, Silva RAR. Meaning of educational actions in nursing consultations for chronic renal clients and relatives. Rev. enferm. UERJ. [Internet] 2018 [cited 2019 Jun 10]; 26:e31921. DOI: https://doi.org/10.12957/reuerj.2018.31921

17. Wu JR, Reilly CM, Holland J, Higgins M, Clark PC, Dunbar SB. Relationship of health literacy of heart failure patients and their family members on heart failure knowledge and self-care. J. fam. nurs. [Internet] 2017 [cited 2019 Jun 10]; 23(1):116-37. DOI: https://doi.org/10.1177/1074840716684808

18. Malta DC, Silva Jr JB. Brazilian strategic action plan to combat chronic non-communicable diseases and the global targets set to confront these diseases by 2025: a review. Epidemiol. serv. saúde. [Internet] 2013 [cited 2019 Jun 10]; 22(1):151-64. DOI: http://dx.doi.org/10.5123/S1679-49742013000100016

19. Mendes EV. O cuidado das condições crônicas na atenção primária à saúde: o imperativo da consolidação da estratégia da saúde da família. Brasília (DF): OPAS; 2012.

20. Chung ML, Lennie TA, Mudd-Martin G, Moser DK. Adherence to the low sodium diet in patients with heart failure is best when family members also follow the diet: a multicenter observational study. J. cardiovasc. nurs. [Internet] 2015 [cited 2019 Jun 
10];30(1):44-50. Available from: https://www.ncbi.nlm.nih.gov/pmc/articles/PMC3999296/

21. Cohen LP, Hummel SL, Maurer MS, López-Pintado S, Wessler JD. Salt taste recognition in a heart failure cohort. J. card. fail. [Internet] 2017 [cited 2019 Jun 10]; 23(7):538-44. DOI: https://doi.org/10.1016/j.cardfail.2017.05.001

22. Barilli SLS, D'Almeida KSM, Trojahn MM, Souza GC, Aliti GB, Rabelo-Silva ER. Knowledge, barriers and attitudes toward dietary sodium in patients with decompensated heart failure. J. nurs. educ. pract. [Internet] 2017 [cited 2019 Jun 10]; 8(1):98-106. DOI: https://doi.org/10.5430/jnep.v8n1p98 